\title{
INTRODUCTION \\ Advancing the care of children with spina bifida, prenatally and postnatally
}

\author{
Erin N. Kiehna, MD, Jeffrey P. Blount, MD, ${ }^{2}$ Catherine McClung Smith, MD, ${ }^{3}$ Eylem Ocal, MD, ${ }^{4}$ and \\ Sandip Chatterjee, MD ${ }^{5}$
}

\begin{abstract}
1'Department of Neurosurgery, Novant Health Neurosciences and Psychiatry Institute, Charlotte, North Carolina; ${ }^{2}$ Children's of Alabama, University of Alabama at Birmingham, Alabama; ${ }^{3}$ Pediatric Neurosurgery, Palmetto Health-University of South Carolina, Columbia, South Carolina; ${ }^{4}$ Department of Neurosurgery, Arkansas Children's Hospital, University of Arkansas for Medical Sciences, Little Rock, Arkansas; and 5epartment of Neurosurgery, VIMS and Park Clinic, Kolkata, India
\end{abstract}

$\mathrm{T}$ HIS issue of Neurosurgical Focus was inundated with a wide variety of papers covering a broad spectrum of clinical care in spinal dysraphism. Topics included diagnosis and maternal considerations, fetal development, and prenatal and postnatal closure techniques, hydrocephalus, Chiari II malformation, and tethered cord management and transitional care.

The incidence of live births of infants with open myelomeningocele (MMC) has been decreasing for more than 2 decades. This is in part due to the implementation of folate fortification of commercially prepared foods and in part due to the increasingly widespread use of screening ultrasound and the subsequent termination of affected pregnancies. The pronounced reduction in open MMC due to folate fortification represents one of the great public health accomplishments of the 20th century. In 1992, the US Public Health Service recommended that all women of childbearing age consume $400 \mu \mathrm{g} / \mathrm{day}$ of folic acid, and in 1998, the US Food and Drug Administration implemented mandatory fortification of grain products with folic acid. Together, these policies reduced the prevalence of neural tube defects by $28 \%$ in the US. Folate fortification can reduce (to rates of 6 affected children/10,000 live births) but not utterly eliminate MMC. Estimated rates for screening and pregnancy termination are unknown.

Regardless of screening or prevention, approximately 1500-2000 births each year in the US are affected by an open MMC, and the incidence elsewhere in the world is orders of magnitude higher. Prior to the mid-20th century (and in many parts of the developing world today), virtually all affected patients died shortly after birth or in early infancy. The development of ventricular shunts and clean intermittent catheterization fundamentally changed this dismal prognosis for survival such that the majority of children born with MMC in the West now live into adulthood. There are many surviving patients from an era in which the incidence of MMC was significantly higher. As a result, in 2019 in the United States of America, there are more adults and young adults than babies and young children alive with MMC. There are several important implications of this: First, the provision of care for affected patients remains critical and must not be lost in the discussion surrounding intrauterine MMC closure (IUMC) issues. Second, there is a great need for transition programs of care and adult care programs in spina bifida. The process of transitioning is poorly understood, and there are only a limited number of transition programs for patients with spina bifida. Third, it is likely that a broader range of neurosurgeons will be called on to provide care for patients with long-term issues related to spinal dysraphism. Patients with MMC are surviving and growing into adulthood. Many struggle, but many more are thriving and making their way more broadly into the world. Neurosurgeons with a variety of backgrounds, training, and practice preferences may be called on to provide care.

In this issue, we present two large series of patients treated predominantly with postnatal closure over the past 20 years and examine the modern incidence and management of postnatal sequelae (Protzenko et al. and Spoor et al.). As hydrocephalus has a significant impact on morbidity and mortality, we then examine the incidence and timing of CSF diversion following birth, noting a trend toward separating the MMC closure from a shunt or third ventriculostomy, without any difference in infection rates (McCarthy et al.). Foss et al. describe the neurobehavioral outcomes of patients with MMC with the hope that early recognition of these potential deficits may lead to earlier intervention and improved outcomes. Finally, Weiss et al. 
provide a comprehensive overview of the effects of MMC on the genitourinary system, which is likely to be helpful to the neurosurgeon struggling to understand the complexities of neuro-urological disease.

The central event to impact clinical care for children with neural tube defects and the spina bifida literature in the past generation was the development of IUMC techniques. While preliminary results of fetal surgery in the 1990s were encouraging, the hallmark publication was the 2011 paper of the Management of Myelomeningocele Study (MOMS). This multicenter $(\mathrm{n}=3)$, prospective randomized controlled trial compared the impact of IUMC at $<26$ weeks' gestation versus conventional postnatal closure on a variety of predetermined primary and secondary endpoints. The original design called for 200 patients to be enrolled but enrollment was halted at 183 because of the documented superiority for IUMC for several endpoints. Intrauterine closure showed clear superiority in the reduction in need for a shunt, reduced severity of hindbrain descent (Chiari II malformation), a more favorable discrepancy between radiological and motor functional level of deficits, and improved short-term motor performance scores. These gains were offset by increased risks of premature delivery (79\% in IUMC treatment arm born before 36 weeks' gestation), spontaneous rupture of membranes, and subsequent uterine dehiscence. The latter factor implicated risks for future pregnancies and mandated subsequent delivery by caesarean section. Fetal death was equal between treatment arms, but the reasons for death differed (premature delivery for IUMC; Chiari II malformation complications in postnatal care).

The promise of these developments was favorably received and resulted in considerable excitement and a rapid expansion of programs offering IUMC. The MOMS trial was and remains the only randomized controlled trial ever published in spinal dysraphism. The study was very well conducted and reported but still had some limitations that remain important in the application of results. The most central of these pertains to the external validity of the trial since the strict inclusion and exclusion criteria of the original prospective randomized controlled trial resulted in a cohort of predominantly nonobese Caucasian pregnant women. Another important concern raised is whether IUMC increases the risk for subsequent tethered cord.

Since the publication of the MOMS trial there has been a vast expansion in the number of centers that offer IUMC. The three original centers standardized the technique but differed in their approach to teaching other centers for subsequent expansion of IUMC programs. Currently, there are at least a dozen centers in the US and another 10 centers throughout the world offering IUMC for MMC. At present, both experience and approach differ considerably among centers.

Great energy and effort have been expended to reduce maternal risk. This has led to the development and increasingly widespread utilization of endoscopic (fetoscopic) techniques. Preliminary (class III) analysis suggests that fetoscopic techniques may reduce maternal morbidity but that the favorable impact of IUMC on hydrocephalus may not be as sustained as it was with the open IUMC technique employed in the MOMS trial. As each of these techniques has evolved, there has been an associated evolution in practice preference with variance in technique among centers. The collective challenge for refinement of techniques in IUMC is that fewer patients are being treated at a larger number of centers with evolving and differing techniques. As a result, attaining sufficient experience clinically and power for studies is increasingly problematic for centers approaching IUMC with an academic commitment. Despite these limitations and challenges, the reported outcomes from IUMC continue to be broadly favorable, and it is likely that the role for IUMC will evolve and become increasingly refined in the coming years. At present, its limited availability and high cost limit major public health impacts within the world regions where demand is greatest.

Many readers may have taken part in one or two studies that surveyed members of the ASPN, ISPN, and/or CNS on their views regarding prenatal closure for MMC (Riley et al. and Gadjradj et al.). While the neurosurgical community at large views prenatal closure favorably, the polling data show that we are exercising due caution. Within this volume, Licci et al. provide the neurosurgical community with a thorough review of maternal and obstetric complications from fetal surgery.

In this issue of Focus, several other papers address current challenges in intrauterine surgery. Lu et al. illustrate that hydrocephalus and need for CSF diversion may develop despite reversal of hindbrain herniation. Cools et al. report that infants who undergo prenatal MMC repair were significantly less likely to be readmitted at 30 days, 90 days, and 1 year owing to a CSF diversion rate of $29 \%$ versus $81 \%$ in the postnatal closure group. Lu et al. analyzed the costs of prenatal closure versus postnatal closure and found that they are equivalent at a year, but when broken down, the costs were higher for mothers in the prenatal cohort and higher for babies in the postnatal cohort.

Much has changed and evolved over the past few decades in treating patients with spinal dysraphism. With improved survivorship, neurosurgeons have an important role to play in transitioning our pediatric patients into adulthood. Patel et al. provide us with a comprehensive review of the spina bifida-related conditions and the challenges involved in the transition of care for patients with spina bifida from infancy into adulthood. Hopson et al. describe a newly developed evidence-based individualized transition plan (ITP) for spina bifida.

This issue of Neurosurgical Focus will hopefully stimulate new discussions regarding the inclusion and exclusion criteria for fetal closure, as well as strategies to continue reducing the morbidity and mortality and improving the quality of life for all patients with spina bifida.

https://thejns.org/doi/abs/10.3171/2019.8.FOCUS19666

\section{Disclosures}

The authors report no conflict of interest.

\section{Correspondence}

Erin N. Kiehna: enkiehna@novanthealth.org. 